\title{
Traduire
}

Revue française de la traduction

$231 \mid 2014$

À table !

\section{Le vin et sa terminologie}

Florence Ludi

\section{OpenEdition}

Journals

Édition électronique

URL : http://journals.openedition.org/traduire/661

DOI : 10.4000/traduire.661

ISSN : 2272-9992

\section{Éditeur}

Société française des traducteurs

\section{Édition imprimée}

Date de publication : 1 décembre 2014

Pagination : 52-59

ISSN : 0395-773X

\section{Référence électronique}

Florence Ludi, « Le vin et sa terminologie », Traduire [En ligne], 231 | 2014, mis en ligne le 01 décembre 2016, consulté le 19 avril 2019. URL : http://journals.openedition.org/traduire/661 ; DOI : 10.4000/ traduire.661 


\section{Le vin et sa terminologie}

\section{Florence Ludi}

\section{Connaître le vin et sa terminologie - comment et pourquoi ?}

"Traductrice spécialisée dans le vin et la gastronomie ? Ça doit être sympa! "C'est vrai : traduire au plus juste - et au plus gourmand - des notes de dégustation de vin, des recettes de cuisine, des articles traitant d'un vignoble ou d'un produit gastronomique, etc., c'est souvent agréable et souvent intéressant.

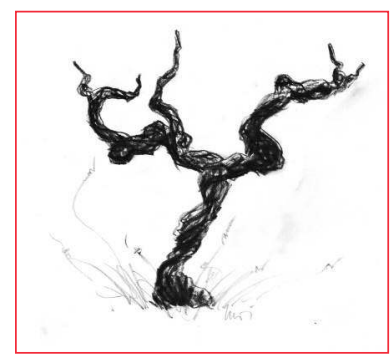

Illustration : Florence Ludi

Cela ne relève pas pour autant des loisirs : au même titre que toute spécialisation professionnelle, le monde du vin et des aliments exige de solides connaissances techniques et terminologiques. D'ailleurs, avant d'être traductrice, j'ai été caviste, animatrice d'ateliers œnologiques, gérante d'un domaine et d'une boutique spécialisée dans les vins du Languedoc, l'épicerie fine et les littératures correspondantes - et j'ai passé deux diplômes en connaissance des vins.

Tout " sympa " qu'il soit, mon métier ne s'improvise pas - mais on peut s'y former. D'une part en suivant des stages idoines(1) et en puisant dans une littérature très fournie(2), d'autre part en participant à des dégustations, en allant voir les producteurs, en se rendant sur des salons, etc.

Le sujet vous met l'eau à la bouche (le vin en est composé à plus de $80 \%$...) ? Découvrez au fil des pages suivantes quelques premières pistes pour qui souhaite aborder la traduction de textes vitivinicoles.

(1) L'auteure de ces lignes propose, par exemple, des formations dans le cadre de la SFT.

(2) Une courte bibliographie est donnée en fin d'article. Je conseille de commencer par l'Encyclopédie du Vin de Jancis Robinson - très technique, mais c'est " la Bible " et elle est parue en plusieurs langues - et par l'Atlas mondial $d u$ vin, de Jancis Robinson et Hugh Johnson - ce dernier est à la fois un grand connaisseur et un merveilleux écrivain du vin. 


\section{Les textes du vin - types les plus fréquents et niveaux de difficulté}

J'ai fait ce que je sais le mieux faire, j'ai lu. Je suis remontée aux sources de l'héritage : Pline l'Ancien, Columelle, Olivier de Serres, philosophes et naturalistes, l'un et l'autre allant alors de pair. [...] Leur langue, sûrement parce qu'elle est plus littéraire que celle de Pierre Galet dans son Précis de viticulture, la bible du viticulteur, me parle.

Catherine Bernard(3)

Dans le domaine du vin, les thèmes traités dans les textes à traduire sont nombreux et les niveaux de difficulté variés, du plus anodin au plus élevé.

Présentation historique. Les caves particulières et coopératives, mais aussi les appellations, etc. se présentent volontiers dans un contexte historique qui leur confère prestige et crédibilité : " nous sommes vignerons depuis dix générations ", " fondée en 1934 pour faire face aux difficultés que connaissaient alors les petits vignerons, notre coopérative... ", " après avoir sillonné le monde comme flying wine maker, notre œnologue de fille a repris les rênes... ", " ayant obtenu l'AOC dès 1947, le vignoble de X... "

Ici, les éventuelles difficultés sont similaires à celles que l'on rencontre dans les textes à caractère touristique : on est confronté à un mélange d'indications très spécifiques, d'ordre historique ou patrimonial, et de contenus parfois très vagues reposant sur des connaissances implicites que le lecteur ne possède souvent pas mais dont l'auteur du texte a une si grande maîtrise qu'il n'en a plus conscience.

Description géographique. En matière de géographie, on peut également avoir affaire à des données très générales comme à des indications très techniques relatives au climat et au microclimat, à l'altitude et à l'orientation des parcelles, etc. Si par exemple " Nos 12 hectares de vignes s'étendent entre Bustince-lriberry et Ispoure " ne pose pas de problème, y compris pour qui n'est pas expert(e) en vins, l'apparente accessibilité d'une phrase comme "Dans le creux de la vallée, à 90 mètres d'altitude, les méandres de la rivière sont un régulateur thermique et une réserve d'eau permanente " cache de réelles difficultés de traduction en raison des raccourcis causaux reposant sur des connaissances techniques implicites : savoir que c'est l'évaporation de l'eau de la rivière qui est un régulateur thermique, savoir à quoi une telle régulation peut être utile, comprendre que les méandres ont ici une fonction purement poétique et que ce ne sont pas eux les régulateurs, etc.

Aspect géologique. C'est pour moi fréquemment l'une des parties les plus difficiles à traduire, d'une part parce que le texte source comporte souvent des données si locales qu'elles ne sont généralement connues que des vignerons du pays et de quelques géologues spécialisés et,

(3) BERNARD Catherine, Dans les vignes, p. 56. 
d'autre part, parce que cet aspect si crucial pour les producteurs n'intéresse généralement pas, en soi, les futurs lecteurs, qu'il s'agisse d'acheteurs, d'importateurs ou de consommateurs. On n'a pas toujours la chance d'avoir à traduire des données telles que

Les éboulis et colluvions limoneux datent de l'ère quaternaire et sont issus de l'érosion des roches par l'action de la pluie ou du gel. Les moûts se caractérisent par un rapport sucre/acidité élevé et une grande puissance tannique.

formulées à la fois clairement et de façon à intéresser le lectorat en indiquant l'influence de ce sol particulier sur les vins issus des parcelles concernées.

Étapes de la production. Les textes consacrés à ces sujets peuvent être succincts et simples ou, au contraire, extrêmement détaillés mais dans tous les cas, ils exigent de la traductrice / du traducteur une bonne connaissance des techniques viticoles et œnologiques. II s'agit principalement de comprendre les processus pour les restituer avec exactitude mais aussi, le cas échéant, de repérer les erreurs qui, au cours des copier-coller successifs, ont pu se glisser dans cette partie si importante de la communication (mention de cépages interdits dans l'appellation concernée, description d'une vinification en rouge alors qu'il s'agit d'un vin blanc, etc.). Ces textes abordent notamment les aspects suivants :

- Les choix culturaux - culture conventionnelle, raisonnée, biologique, biodynamique, période de conversion, etc.

- Les choix viticoles - de la sélection des porte-greffes (répondant par exemple au doux nom de S04 ou 44-53 M), des cépages et de leurs clones (carignan CFC8, riesling 1089, etc.) aux types de vendanges - diurne, nocturne, mécanique ou manuelle, en caissettes ou camions réfrigérés, en passant par le type de taille et de palissage, les travaux d'ébourgeonnage, d'épamprage, le choix de l'enherbement (complet ou partiel) ou du type de désherbage (mécanique ou chimique), les vendanges en vert, etc.

- Les étapes de la vinification selon des méthodes œnologiques traditionnelles ou novatrices. Celles-ci comptent, selon le résultat visé - c'est-à-dire un vin plus ou moins vif, rond, fin, gras ou tannique, un vin plaisir ou un vin de garde, etc. -, divers procédés incontournables ou facultatifs : débourbage, enzymage, éraflage, filtration, foulage, levurage, remontage, pressurage, soutirage, sulfitage, etc.

Décrypter et traduire le sens de

Nos vins sont vinifiés à la bourguignonne, éraflage et encuvage après un passage sur la table de tri, pas de thermovinification; fermentation avec les levures indigènes, remontages journaliers ; les temps de cuvaisons sont fonction du millésime.

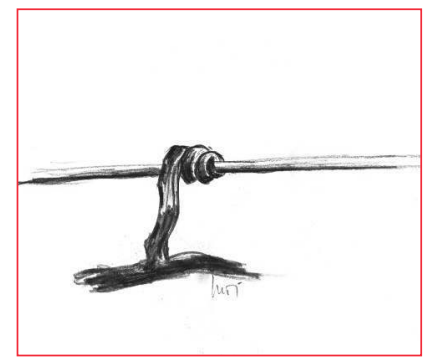

Illustration : Florence Ludi 
exige non seulement, comme cela apparaît au premier coup d'œil, de solides connaissances techniques, mais aussi culturelles, car "vinifié à la bourguignonne ", pour ne pas être un terminus technicus, ne s'en réfère pas moins à une méthode bien précise, la fermentation en fût avec bâtonnage (c'est-à-dire remise en suspension des lies tombées au fond) régulier, qu'une traduction mot à mot, pour n'être pas franchement fausse, ne rendrait pas de façon adéquate.

- Un éventuel élevage - en cuve, en fût ou en bouteille.

- Le conditionnement des vins : forme et couleur des bouteilles ou choix d'autres contenants, matériau et qualité des bouchons, contre-étiquette ou non, type de carton ou de caisse en bois, etc.

Fiche technique. Elle contient généralement une mention plus ou moins détaillée des aspects ci-dessus, auxquels viennent s'ajouter des notes de dégustation assorties de conseils d'accords mets-vins ainsi que, le cas échéant, des précisions concernant le millésime, les récompenses décernées au vin en question, etc. La principale difficulté réside ici dans les notes de dégustation (cf. ci-après).

Notes de dégustation. Les termes se référant à la dégustation du vin, c'est-à-dire à son appréciation organoleptique, n'existent pas tous dans l'ensemble des langues de la civilisation occidentale (pour parler de ce dont j'ai une certaine connaissance), malgré une longue tradition de production et de consommation. Ainsi, la notion d'" attaque ", la première sensation en bouche, est-elle par exemple absente de l'allemand d'Allemagne, où le sujet est majoritairement éludé, ou traduit par le très flou anfangs (mais elle existe en allemand de Suisse où l'on utilise le terme d'Ansatz). Concernant les traductions vers ou depuis des langues se rattachant à des cultures très éloignées, je ne peux qu'imaginer les difficultés rencontrées par mes consœurs et confrères. À titre d'exemple, l'Asie connaît un cinquième goût, l'umami - que l'on peut traduire par "savoureux " -, tandis que nous n'en avons que quatre - acide, salé, sucré, amer. Mais les différences susceptibles d'exister entre deux pays pourtant culturellement proches à première vue peuvent exiger une réflexion métaconceptuelle et une adaptation ou une précision de certains termes. Ainsi, aux États-Unis, un vin dont l'étiquette mentionne

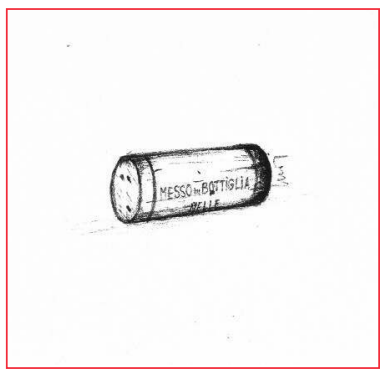

Illustration : Florence Ludi
"Cabernet-Sauvignon 2010 " peut-il contenir légalement jusqu'à $25 \%$ d'un autre cépage et/ou d'un autre millésime. Ceci étant interdit en France, une précision peut s'avérer nécessaire lors de la traduction.

Pour les accords mets-vins, on se heurte aux multiples spécificités culinaires qui exigent parfois une importante adaptation aux habitudes gastronomiques des lecteurs, voire une réécriture pure et simple. Cela s'imagine aisément pour le cassoulet ou la tartiflette, mais concerne également les charcuteries, les 
fromages, la préparation des légumes et des (sauces de) salades, etc. Ainsi de "salade de mâche fraîche au lard fumé et aux Kracherle, ces croûtons badois revenus au beurre ", de Remouladensauce qui devient par ex. "sauce gribiche " (et pas rémoulade !), bien que les deux recettes ne soient pas exactement identiques, de " gardiane de taureau " qui se mue par exemple, pour un lectorat allemand qui ne mange pas de taureau, en " pot au feu de bœuf au vin rouge ", d'، andouillette " ou de " matjes » intraduisible, etc.

Généralement, les textes du vin associent plusieurs des thèmes présentés ci-dessus. Ainsi, le site internet d'un producteur, par exemple, comporte à la fois un aperçu historique de la cave, une présentation géologique et géographique du vignoble, des précisions sur les méthodes culturales et les techniques œnologiques, la fiche technique de chaque vin, etc. Les degrés de technicité et de difficulté peuvent donc être très variables au sein d'une seule et même traduction.

\section{Aspects spécifiquement terminologiques}

Depuis que ma géniale payse, la grande Colette, n'est plus, où trouverez-vous une fille qui sache comme je le sais d'enfance ce qu'étaient "sombrer " une vigne, la "rueller", la "biner ", l'" écouler " [...] ?

Marie Noël(4)

La compréhension et le maniement de la terminologie du vin impliquent plusieurs exigences, dont la principale est commune à toutes les spécialisations : il s'agit de la connaissance et de la compréhension des processus techniques particuliers au domaine concerné. Dans le secteur du vin, ce sont les connaissances viticoles et œnologiques, mais aussi celles relatives au conditionnement, à la mise en marché, à la législation spécifique, à la dégustation, sans oublier ce qui relève de domaines adjacents tels que la tonnellerie, la cristallerie, etc.

II existe par ailleurs une série de points terminologiques susceptibles de présenter des problèmes de compréhension ou d'usage :

- termes polysémiques tels que " chai " ou " cru ";

- termes phonologiquement ou graphiquement similaires mais qui désignent des signifiés partiellement ou complètement différents, par ex. "la vendange " et " les vendanges " ;

- termes fréquemment employés à mauvais escient comme "terroir " que beaucoup substituent volontiers à " territoire " ou à " sol ", soit par ignorance, soit parce qu'ils trouvent que cela " sonne " plus prestigieux ;

(4) Citée dans PIVOT Bernard, Dictionnaire amoureux du vin, p. 279. 
- variantes régionales du vocabulaire, de la vigne notamment.

En outre, le langage vitivinicole comporte des synonymes qu'il est intéressant de connaître pour enrichir son style (par exemple " cep " / " souche " / " pied " / " plant de vigne ").

Enfin, il est important de maîtriser les registres linguistiques desquels relèvent les termes rencontrés, par exemple les registres respectifs de " tonneau " et de " fût " ou de "bouteille " et de " col ", etc.

\section{Déguster le vin et le décrire : learning by doing}

On pourrait croire, tant les arômes sont nombreux dans l'universelle variété des vins, que ceux-ci sont un condensé de la Création, le réceptacle magique du foisonnement et de l'hétérogénéité de la nature. Tous les bons vins sont des énigmes plus ou moins complexes. C'est pourquoi la dégustation - de la muqueuse olfactive du nez à la mémoire olfactive du cerveau - est une science pour les professionnels et un jeu pour les amateurs, pour tous une passionnante quête d'identité.

Bernard Pivot(5)

Pour qui veut aborder professionnellement le domaine du vin, il est indispensable d'apprivoiser la dégustation. En effet, déguster permet de s'initier aux techniques professionnelles d'évaluation visuelle, olfactive et gustative, de découvrir concrètement, d'un vin à l'autre, les différences de couleur, de viscosité, de bouquet, de matière, de persistance aromatique, etc. et de commencer à s'exercer à faire usage du vocabulaire propre à la dégustation. Car déguster, c'est non seulement percevoir, mais également nommer aussi précisément que possible ses perceptions sensorielles. C'est une interaction intense entre les sens et le cerveau (mémoire, zones du langage) : on est au cœur du sujet.

Certes, ce n'est pas un exercice facile car, en dehors des professionnels du vin, rares sont les personnes ayant déjà fait l'analyse structurée d'un vin. Certaines, même, n'en boivent pas ce qui n'est d'ailleurs pas un problème, la dégustation faisant appel à des sens que nous utilisons tous, ainsi qu'à notre mémoire et à notre capacité à reconnaître et à nommer ce que perçoivent nos sens. Pas facile, donc, mais instructif et, une fois la première timidité passée, c'est le côté ludique qui l'emporte et, avec lui, le plaisir de découvrir que tout le monde peut déguster du vin et que l'expérience de la vie permet à chacun(e) d'établir des distinctions, de percevoir des arômes, etc. : c'est une "simple " question d'entraînement.

(5) PIVOT Bernard, Dictionnaire amoureux du vin, p. 30. 
Au cours de mes formations, la terminologie de la dégustation est ainsi introduite par l'expérience (une méthode didactique qui a fait ses preuves) tout en étant expliquée et commentée au niveau métalinguistique : ces moments particulièrement conviviaux sont donc aussi riches en apprentissage que les parties de séminaire pur ou de visite de terrain - et permettent de terminer chaque journée de façon à la fois active et détendue.

\section{En guise de conclusion}

Chère lectrice, cher lecteur, j'espère vous avoir fait toucher du doigt (ou du palais) à quel point une spécialisation dans le domaine du vin, pour être liée au plaisir et à la convivialité, n'en est pas moins extrêmement exigeante. Et que l'on n'aborde pas un texte vitivinicole à la légère, sans connaissances préalables.

J'espère aussi avoir éveillé en vous la gourmandise d'en savoir plus et de découvrir cet univers d'une incroyable richesse, synergie entre les trésors de la Terre, l'intelligence et le savoir-faire de l'homme (et, de plus en plus, de la femme) et la joie d'être, tout simplement.

Et je me réjouis d'avance de vous rencontrer, un jour, à l'occasion d'une formation ou autour d'un verre de bon vin.

ludi.traductions@orange.fr

P.S. : On me demande comment je suis " tombée dans le vin ". En fait - et si je fais abstraction de ce que mes grands-parents paternels faisaient leur propre vin et de ce que la lignée maternelle était bourguignonne et comptait plusieurs membres du Tastevin -, par pur hasard. Alors que mon engagement comme tutrice en DEUG de linguistique à la Freie Universität de Berlin tirait à sa fin, au contraire de mes études qu'il me fallait urgemment trouver un moyen de financer, j'ai appris d'une de mes étudiantes qui vendait du vin français que son chef cherchait une nouvelle collaboratrice. II m'a engagée : j'étais " tombée dedans " !

À l'aspect alimentaire du job s'est bientôt ajouté un intérêt personnel et cinq ans plus tard, maîtrise de linguistique en poche, j'animais des séminaires et des dégustations à thème. De plus en plus passionnée par le sujet, j'ai repris des études d'œnologie en Bourgogne (le hasard, vous dis-je...). Après avoir été stagiaire dans un syndicat d'appellation, exercé comme commerciale export puis comme gérante d'un domaine dans le Languedoc, j'ai créé en 2005 un bureau de traduction spécialisé dans les domaines du vin et de la gastronomie. Parallèlement à mon activité de traduction et de rédaction, je propose un éventail de formations professionnelles autour du vin et de la terminologie vitivinicole. 


\section{Bibliographie}

BERNARD Catherine, 2011, Dans les vignes, Chroniques d'une reconversion, Arles, Éditions du Rouergue.

JOHNSON Hugh et ROBINSON Jancis, 2014, L'Atlas du vin, Paris, Flammarion.

PIVOT Bernard, 2006, Dictionnaire amoureux du vin, Paris, Plon.

ROBINSON Jancis, 1997, L'Encyclopédie du vin, Paris, Hachette.

Traductrice libérale et d'édition depuis 2005, Florence Ludi est spécialisée dans les domaines du vin et de l'alimentation. Elle a notamment traduit aux Éditions Thierry Souccar Boire mieux pour vivre vieux (2009, titre original The Wine Diet, 2007) du professeur Roger Corder. Description détaillée sur http://vinquebec.com/node/7111

Depuis 1999, Florence Ludi anime des ateliers de dégustation, des ateliers d'initiation à l'univers du vin ainsi que, depuis 2007, des formations professionnelles sur le vin et sa terminologie, notamment dans le cadre de la SFT.

Elle est titulaire d'une Maîtrise de Linguistique en langues et civilisation germaniques (Paris IV Sorbonne), d'un Mastère spécialisé Connaissance et commerce international des vins (AgroSup Dijon) et de I'International Higher Certificate in Wines \& Spirits (Niveau 3 du Master of Wines, Wine \& Spirit Education Trust Londres). 